COMENTARIO

\title{
Utilización de los actinomicetos en procesos de biofertilización
}

\section{Use of actinomycetes in processes biofertilization}

$\begin{array}{ll}\text { Presentado: } & 27 / 08 / 2009 \\ \text { Aceptado: } & 28 / 11 / 2009 \\ \text { Publicado online: } & 12 / 01 / 2010\end{array}$

\section{Marcela Franco-Correa}

Laboratorio de Microbiología Ambiental y de Suelos, Grupo de Biotecnología Ambiental e Industrial. Departamento de Microbiología, Pontificia Universidad Javeriana. Bogotá, Colombia. Email Marcela Franco: franco@javeriana.edu.co
El mal uso agrícola de los suelos, que desafortunadamente ocurre en diferentes zonas cultivables, ha ido intensificando los problemas de fertilidad en los mismos. Particularmente, el uso intensivo de los fertilizantes y productos fitosanitarios químicos ha conllevado a elevar los costos de producción de muchos alimentos y otros derivados de la agricultura haciéndolos poco competitivos en el mercado. Los manejos agrícolas más sostenibles preconizan la disminución de agroquímicos, como alternativa al uso de estos compuestos, estrategias que cada día cobran más interés. En relación con los manejos sostenibles en agricultura, la posibilidad de usar técnicas basadas en el manejo de microorganismos que usualmente viven asociados a las plantas, ocurre en virtud de las acciones positivas que se sabe realizan. En efecto, determinados microorganismos que se desarrollan en asociación con las raíces de las plantas, en la llamada rizosfera, están involucrados en la promoción de crecimiento vegetal mediante un amplio rango de actividades. Estas acciones incluyen la estimulación de los ciclos biogeoquímicos de los nutrientes, mejora de la salud de la planta y la calidad estructural del suelo, factores claves de la fertilidad del mismo.

Como consecuencia de lo anterior se hace necesario entender los diferentes factores que regulan la actividad microbiana relacionada con el mantenimiento de la fertilidad del suelo y la nutrición vegetal. Es así que muchas investigaciones han intentado enfocarse en la búsqueda de microorganismos con habilidad para mejorar el enraizamiento, disolver el fósforo mineral no asimilable por la planta, fijar nitrógeno de la atmósfera, producir sideróforos, interactuar con otros microorganismos, entre otras actividades.

En este contexto, es de reseñar los pocos estudios publicados sobre los actinomicetos, bacterias conocidas por desarrollar diversas actividades en el ecosistema, tales como el mejoramiento de la estructura del suelo y producción de compuestos bioactivos con actividad antagonista contra microorganismos patógenos, siendo los principales productores de antibióticos. Particularmente, se han descrito actividades que pueden catalogar a los actinomicetos como rizobacterias promotoras del crecimiento vegetal: PGPR (del inglés Plant Growth Promoting Rhizobacteria). Es así que cabe resaltar como la línea de investigación titulada: Microorganismos con Potencial Agroindustrial, ha sido pionera en el avance de este tema (Franco-Correa 1999; Franco-Correa 2001; Orjuela et al. 2003; Martínez et al. 2003; Márquez et al. 2003; Franco-Correa 2008).

Dado que en investigaciones de ecología microbiana en la rizosfera es importante considerar la interacción entre los diferentes microorganismos, especialmente entre aquellos que benefician a las plantas y pueden mejorar la productividad agrícola, se debe incluir a los hongos formadores de micorrizas arbusculares. La mayoría de las especies de plantas silvestres y cultivadas forman micorrizas, siendo las llamadas micorrizas arbusculares (MA) las más comunes y, concretamente, las que forman las plantas de interés agronómico, entre ellas cereales, leguminosas, hortícolas y frutales. Debido a los efectos de las MA, enfocados hacia la biofertilización, fitoestimulación y bioprotección de los cultivos, se acepta que el manejo apropiado de esta simbiosis puede permitir una reducción significativa de fertilizantes y fungicidas químicos, aspectos claves en una producción sostenible en agricultura, con los consiguientes beneficios ecológicos y económicos.

Teniendo en cuenta los antecedentes citados se han realizado dentro del grupo de Biotecnología Ambiental e Industrial, una gran cantidad de investigaciones para incrementar los conocimientos sobre actinomicetos, bacterias comunes en el suelo y que ayudan a mantener el equilibrio ecológico en zonas con una alta explotación agrícola. La finalidad ha sido estudiar la posibilidad su posible utilización como biofertilizante, de ahí el interés de caracterizar estas bacterias como PGPR dilucidando su comportamiento en cuanto a sus interacciones con las MA para llegar a concluir la presencia de relaciones sinérgicas benéficas entre estos dos grupos microbiológicos (Franco-Correa 2008).

Para poder reunir varios aspectos relacionados con la promoción de crecimiento e interacción con hongos MA se han trabajado algunas plantas como trébol, frijol y clavel, para luego continuar en un futuro, las pruebas en cultivos de alto interés agrícola, particularmente en Colombia.

El estudio de la diversidad genética en microorganismos como los actinomicetos tiene gran importancia en la sostenibilidad de los suelos colombianos, para poder en un futuro cercano presentarlos como un recurso clave a nivel de biotecnología agrícola, y así mismo, poder determinar los diferentes patrones que presentan estos microorganismos y su interacción con los hongos MA y las plantas que permitan utilizarlos para el seguimiento de los efectos del cambio ambiental global.

A inicios del siglo XX, Hiltner introdujo el término rizosfera para describir la zona del suelo afectada por el desarrollo de las raíces, las cuales inducen la proliferación de los microorganismos. Las actividades metabólicas de tales poblaciones estimuladas, tanto desde el punto de vista cualitativo como cuantitativo, en la rizosfera son de vital importancia para el desarrollo de las plantas (Azcón-Aguilar et al. 2002). El incremento de la actividad microbiana en la rizosfera, es ejercido por el suministro de compuestos orgánicos (solubles o insolubles) que aportan los exudados radicales y otros materiales, en general, residuos vegetales o microbianos, recibe el nombre de efecto rizosférico. 
El término rizobacteria refiere a la capacidad de dichos microorganismos para colonizar preferencialmente las interfases suelo-raíz, donde mantienen poblaciones de individuos a un nivel que permite su efectividad (Kloepper et al. 1991; Barea et al. 2005).

Los hongos micorrizógenos y las bacterias fijadoras de $\mathrm{N}_{2}$ son los componentes más destacados entre los simbiontes mutualistas. Los hongos de la micorriza, una vez que colonizan de forma biotrófica la raíz, desarrollan un micelio externo que la conecta con los microhábitats del suelo tanto rizosférico como, fundamentalmente, el no-rizosférico, lo que les permite desarrollar actividades tales como el ciclado de nutrientes ( $\mathrm{P}$ y $\mathrm{N}$ fundamentalmente), protección frente a estreses bióticos y abióticos, conservación del suelo (formación de agregados), establecimiento de las plántulas, reguladores de la sucesión vegetal, entre otros. Las bacterias simbióticas fijadoras de $\mathrm{N}_{2}$ (Rhizobium, Frankia y cianobacterias) efectúan su relevante función en la rizosfera de plantas de interés tanto en agrosistemas como en ecosistemas naturales (Barea y Olivares 1998).

Los actinomicetos representan un grupo ubicuo de microorganismos ampliamente distribuido en ecosistemas naturales y tienen gran importancia en la participación de la degradación de materia orgánica, además de ciertas propiedades fisiológicas que los hacen particulares (Ghanem et al. 2000). En un principio los actinomicetos se incluyeron entre los hongos porque su morfología y desarrollo presentaban gran similitud, dotados de un micelio verdadero; debido a esto se les denominó "hongos radiados". Sin embargo, hoy en día, y dado su carácter procariótico, se sustenta muy bien su clasificación como bacterias (Koneman 2001; Prescott 2002).

Estos microorganismos resultan ser abundantes en suelos, tanto o más que las mixobacterias (Ben-Omar et al. 1997), sin embargo, también se encuentran en ambientes acuáticos, dulces y marinos (Leiva et al. 2004). Dentro de sus características particulares presentan un olor típico a suelo húmedo por la producción de un metabolito llamado geosmina, adicionalmente presentan una actividad metabólica alta, producen terpenoides, pigmentos y enzimas extracelulares con las que son capaces de degradar la materia orgánica de origen vegetal y animal (Ezziyyani et al. 2004). Como la gran mayoría de bacterias que son abundantes en suelo, presentan un importante papel ecológico en el mismo (Ben-Omar et al. 1997).

En el suelo se encuentran en casi todos los tipos y bajo condiciones extremas disminuyen levemente la concentración de la población. Su número varía en gran proporción según el caso, pero es común encontrarlos en suelos fértiles con concentraciones de $10^{6} \mathrm{UFC} \cdot \mathrm{g}^{-1}$ de suelo seco. Por lo general se aíslan cepas de actinomicetos en la superficie del suelo y en profundidades entre 2 y $15 \mathrm{~cm}$, más allá de esta profundidad disminuye la cantidad de éstos. El tamaño de la comunidad depende del tipo del suelo, particularmente de algunas de las características físicas, del contenido de materia orgánica y del $\mathrm{pH}$ del medio ambiente (Tate 2000).

Los principales géneros que se aíslan a partir de suelos son Nocardia, Streptomyces y Micromonospora, que pueden estar presentes como conidias o como hifas vegetativas (Martin 1981). Sin embargo, los métodos de aislamiento convencionales muestran que el $95 \%$ de los actinomicetos aislados a partir de suelo pertenecen al género Streptomyces (Lacey 1973). El uso de técnicas moleculares para la detección de estas bacterias en diferentes ambientes ha mostrado la presencia de organismos de la clase Actinobacteria que no han podido ser aislados por técnicas tradicionales de cultivo. Ciertos de estos organismos no han sido identificados específicamente, y la posición filogenética de algunos subgrupos muestra que su divergencia corresponde con la de los géneros tradicionales de actinomicetos, indicando una estructura rica en organismos de esta clase y no cultivable en suelos (Rheims et al. 1999).

Algunos géneros han sido reportados como fijadores de nitrógeno atmosférico, como Frankia y algunas cepas pertenecientes a las familias Thermomonosporaceae y Micromonosporaceae (Valdés et al. 2005; Franco-Correa 2008).

Los actinomicetos también han sido descritos como agentes de biocontrol por la capacidad de producir enzimas biodegradativas como quitinasas, glucanasas, peroxidasas y otras, involucradas en el papel del micoparasitismo que llevan a cabo estos microorganismos (Franco-Correa 1999; Tokala et al. 2002; Márquez et al. 2003). El género Streptomyces ha sido descrito como colonizador de la rizosfera, capaz de ejercer biocontrol sobre hongos fitopatógenos, producir sideróforos, sustancias promotoras del crecimiento vegetal in vitro, promover la nodulación y ayudar a los bacteriodes de Rhizobium a la asimilación del hierro en la fijación de nitrógeno en leguminosas, lo cual contribuye indirectamente a la promoción de crecimiento vegetal (Tokala et al. 2002).

Las bacterias que colonizan la raíz y su zona de influencia (suelo rizosférico) son denominadas "rizobacterias" (Kloepper, 1994 y 1996). La literatura científica que describe las actividades de estas bacterias es amplia (Kloepper et al. 1991, Hass 1991; O'Gara et al. 1994; Welller y Thomashow 1994; Glik 1995; Van de Broek y Vanderleyden 1995; Bashan y Holguin 1998; Barea 2000; Gutierrez-Mañero et al. 2001, 2002; Probanza et al. 2002; de Boer et al. 2003; Persello-Cartieaux et al. 2003; Barea et al. 2004; Zahir et al. 2004; Gamalero et al. 2004).

El concepto de mycorrhiza helper bacteria (MHB) fue introducido en un Tansley Review: Helper Bacteria: una nueva dimensión de la simbiosis micorricica (Garbaye 1994), que ha conllevado a nuevas investigaciones en el sistema modelo planta-hongo, en cuanto al significado de estas bacterias que promocionan la formación de las micorrizas y ocasionan muchos efectos fisiológicos de la interacción mutualista. El concepto de $\mathrm{MHB}$ es genérico, este depende del tipo de simbiosis micorrícica pero no de la posición taxonómica de la bacteria. En relación con las MA, han sido descritos muchos ejemplos (Abdel-Fattal y Mohamedin 2000; Barea et al. 2005; Artusson et al. 2006). Ciertos actinomicetos (Rhodococcus, Streptomyces y Arthrobacter) han sido identificados como bacterias que se asocian al micelio de hongos ectomicorrícicos (Burke et al. 2006), y solo hay una referencia de Streptomyces coelicolor asociado a Glomus intraradices en cultivos de sorgo (Abdel-Fatah y Mohamedin 2000).

Aunque hay numerosos estudios referentes a las interacciones de los hongos MA y rizobacterias, asociaciones entre MA y actinomicetos se conocen escasamente, por lo que se requieren investigaciones que permitan la confirmación experimental adicional sobre actividades y mecanismos relacionados. Las investigaciones en micorrizas deben por lo tanto esforzarse ha- 
cia una comprensión mejorada de los mecanismos funcionales involucradas en las interacciones en la micorrizosfera, para poder desarrollar la biotecnología ad hoc que permita aplicar las combinaciones optimizadas de microorganismos como inoculadores eficaces dentro de sistemas sostenibles de la producción vegetal (Andrade et al. 1997; Artursson et al. 2006).

Los resultados encontrados en la investigación realizada dentro del grupo, apoyan, por tanto, interacciones tanto entre planta-microorganismo, ya sean actinomicetos u hongos MA, (rizosfera), como MA-actinomicetos (micosfera), para desarrollar una autentica micorrizosfera (Barea et al. 2005) que promueve el crecimiento y nutrición de las plantas (Franco-Correa 2008).

Con base en la reseña elaborada y en las investigaciones realizadas en el grupo, se puede concluir que los actinomicetos han demostrado ser unos microorganismos promisorios en la biofertilización sin afectar los otros microorganismos rizosféricos, manteniendo el equilibrio rizosférico, uno de los principales objetivos en los cuales se basa la investigación en Microbiología de Suelos y la Interacción Microorganismo-Planta.

\section{Literatura citada}

Abdel-Fatah G.M. \& A.H. Mohamedin. 2000. Interactions between a vesicular-arbuscular mycorrhizal fungus (Glomus intraradices) and Streptomyces coelicolor and their effects on sorghum plants grown in soil amended with chitin of brawn scales. Biol Fertility Soils 32:401-409

Andrade G., K.L. Mihara, R.G. Linderman \& G.J. Bethlenfalvay. 1997. Bacteria from rhizosphere and hyphosphere soils of different arbuscular mycorrhizal fungi. Plant and Soil, 192:71-79

Artursson V., R.D. Finlay \& J.K. Jansson. 2006. Interactions between arbuscular mycorrhizal fungi and bacteria and their potential for stimulating plant growth. Environmental Microbiology 8: 1-10

Azcón-Aguilar C., J. Palenzuela, A. Roldan, et al. 2002. Analysis of the mycorrhizal potential in the rizosphere of representative plant species from desertification-threatened Mediterranean shrublands. Applied Soil Ecology. 21: 1-9

Barea J.M. 2000. Rhizosphere and mycorrhiza of field crops. En: Biological Resource Management: Connecting Science and Policy. (Balázs E, Galante E, Lynch JM, Schepers JS, Toutant JP, Werner D, Werry PA Th J, eds) Berlin, Heidelberg, New York: INRA Editions, Springer-Verlag, $110-125$

Barea J.M. \& J. Olivares. 1998. Manejo de las propiedades biológicas del suelo. En: Agricultura sostenible. RM. Jiménez-Díaz, J. Lamo de Espinosa (Eds.) pp. 173-193. Mundi prensa. Madrid

Barea J.M., R. Azcón \& C. Azcón-Aguilar. 2004. Mycorrhizal fungi and plant growth promoting rhizobacteria. En: Plant Surface Microbiology (A. Varma, L. Abbott, D. Werner, R. Hampp Eds) Springer-Verlag, Heidelberg, Alemania. pp. 351-371

Barea J.M., M.J. Pozo \& C. Azcon-Aguilar. 2005. Microbial cooperation in the rizosphere. J. Expe: Bot. 56: 1778-1784

Bashan Y. \& G. Holguin. 1998. Proposal for the division of plant growth-promoting rhizobacteria into two classifications: biocontrol-PGPB (plant growth-promoting bacteria) and PGPB. Soil Biology and Biochemistry, 30:1225-1228

Ben-Omar N., M.L. Merroun, J.M. Arias Peñalver \& M.T. Gonzalez Muñoz. 1997. Comparative heavy metal biosorption study of Brewery yeast and Myxococcus xanthus biomass. Chemosphere. 35(10):2217-2283
Burke D.J., A.M. Kretzer, P.T. Rygiewicz \& M.A. Topa. 2006. Soil bacterial diversity in a loblolly pine plantation: Influence of ectomycorrhizas and fertilization. FEMS Microbiology Ecology 57:409-419

De Boer W., P. Verheggen, P.J.A. Klein Gunnewiek, et al. 2003. Microbial community composition affects soil fungistasis. Appl. Environ. Microbiol. 69:835-844

Ezziyyani M., C. Pérez, M. Requena, et al. 2004. Evaluación del biocontrol de Phytophthora capsici en pimiento (Capsicum annuum L.) por tratamiento con Burkholderia cepacia. Anales de Biología 26:61-68

Franco-Correa M. 1999. Aislamiento, Caracterización y Evaluación de Actinomycetes inhibidores de algunos hongos fitopatógenos. Tesis de Maestría en Microbiología, Departamento de química, Instituto de Biotecnología, Universidad Nacional de Colombia. págs. 86

Franco-Correa, M. 2001. Los actinomycetes: Potencial biotecnológico y agroindustrial. Memorias del curso postcongreso del II Congreso Peruano de Biotecnología y Bioingeniería. pp. 65

Franco-Correa, M. 2008. Evaluación de caracteres PGPR en actinomicetos e interacciones de estas rizobacterias con hongos formadores de micorrizas. Tesis Doctoral, Director: Dr. José Miguel Barea N. Departamento de Fisiología Vegetal. Facultad de Ciencias. Universidad de Granada. pp. 266

Gamalero E., A. Trotta, N. Massa et al. 2004. Impact of two fluorescent pseudomonads and an arbuscular mycorrhizal fungus on tomato plant growth, root architecture and $\mathrm{P}$ acquisition. Mycorrhiza 14:185-192

Garbaye J. 1994. Helper bacteria: a new dimesion to the mycorrhizal symbiosis. New Phytologist 128(2): 197-210

Ghanem N.B., S.A. Sabry, Z.M. El-sherif, et al. 2000. Isolation and Enumeration of marine Actinomycetes from seawater and sediments in Alexandria. Applied and Environmental Microbiology. 46:105-111

Glick BR. 1995. The enhancement of plant growth by free-living bacteria. Can J Microbiol 41:109-117

Gutiérrez-Mañero F., B. Ramos-Solano, A. Probanza, et al. 2001. The plant-growth-promoting rhizobacteria Bacillus pumilus and Bacillus licheniformis produce high amounts of physiologically active gibberellins. Physiol Plant 111:206-211

Gutiérrez-Mañero F.J., B. Ramos, J.A. Lucas Garcia, et al. 2002. Systemic induction of terpenic compounds in D. lanata. J Plant Physiol 160:105-113

Hass D., C. Keel, J. Laville, et al. 1991. Secondary metabolites of Pseudomonas fluorescens strain CHA0 involved en the suppression of root diseases. En: Advances en Molecular Genetics of Plant.Microbe interactions, Vol. I H. Hennecke and D.P.S. Verma (eds). Kluwer Academic Publishers, Dordrecht, The Netherlands, pp.450-456

Kloepper J.W. 1994. Plant growth promoting rhizobacteria (other system). En: Azospirillum/Plant Association (Y. Okon, Ed.), CRC Press, Boca Raton, Florida, USA. pp. 135-166

Kloepper J.W. 1996. Biological control agents vary in specificity for host, pathogen control, ecological habitat and environmental conditions. Bio. Sci. 46:406-409

Kloepper J.W., R.M. Zablotowicz, E.M. Tipping, R. Lifshitz. 1991. Plant growth promotion mediated by bacterial rhizosphere colonizers. En: The rhizosphere and plant growth, Keister DL, Cregan PB (eds). Kluver acadeic Publishers, Netherlands, pp 315-326

Koneman E.W. 2001. Diagnóstico Microbiológico: Texto y Atlas a Color. Quinta Edición. Médica Panamericana. Buenos Aires, Argentina. pp. 10-16, 75-82 
Lacey J. 1973. Actinomycetes in soils, composts and fodders in Actinomycetales: Characteristics and Practical Importance, G Sykes, FA Skinner (Eds) Academic Press, London, pp. 231-251

Leiva S., M. Yáñez, L. Zaror, et al. 2004. Actividad Antimicrobiana de actinomycetes aislados desde ambientes acuáticos del sur de Chile. Revista médica de Chile.132:151-159

Márquez M., M. Martínez, \& M. Franco. 2003. Aislamiento de Trichoderma sp. y actinomycetes a partir de suelos de clavel (Dianthus caryophyllus) y evaluación de su capacidad antagónica in vitro sobre Fusarium oxysporum f. sp. dianthi. Revista Agronomía Colombiana. XIX (1-2): 81-88

Martin A. 1981. Introducción a la microbiología del suelo. AGT editores. Ciudad de México. pp.158

Martínez, M., M. Franco, \& L. Díaz. 2003. Memorias del Curso de Microbiología Aplicada a la Agricultura. Educación Continuada, Facultad de Ciencias, Pontificia Universidad Javeriana. pp. 53

O'Gara F., D.N. Dowling \& B. Boesten. 1994. Molecular Ecology of Rhizosphere Microorganisms. Weinheim, Germany: $\mathrm{VCH}, 173$

Orjuela P., A. Sandón, M. Franco, \& M. Martínez. 2003. Evaluación de cepas antagónicas de actinomicetos y de Trichoderma spp. aislados a partir de suelos de cultivos de arroz (Oryza sativa) para el control de Rhizoctonia solani. Revista Mundo Microbiológico. Asociación Colombiana de Microbiología. 2( 2): 9-14

Persello-Cartieaux F., L. Nussaume \& C. Robaglia. 2003. Tales from the underground: molecular plant-rhizobacteria interactions. Plant Cell and Environment 26:189-199

Prescott, L.M. 2002. Microbiology. 5th ed. Editorial McGraw-Hill. Boston, Massachusetts, USA. pp. 524-528
Probanza A., G.J.A. Lucas, P.M. Ruiz, et al. 2002. Pinus pinea L. seedling growth and bacterial rhizosphere structure after inoculation with PGPR Bacillus (B. licheniforme CECT 5106 and B. pumilusCECT 5105). Applied Soil Ecology 20:75-84

Rheims H., C. Spróer, F.A. Rainey \& E. Stackebrandt. 1999. Molecular biological evidence for the occurrence of uncultured members of the Actinomycete line of descent in different environments and geographical locations. Microbiology. $142: 2863-2870$

Tate R.L. 2000. Soil Microbiology (second ed.), Wiley, New York, pp. $47-56$

Tokala K., C. Strap, D. Jung, et al. 2002. Novel plant-microbe rhizosphere interaction involving Streptomyces lydicus WYEC108 and the pea plant (Pisum sativum). Applied and Environmental Microbiology. 68:2161-2171

Valdés M., N. Perez, P. Estrada, et al. 2005. Non-Frankia Actinomycetes Isolated from Surface-Sterilized Roots of Casuarina equisetifolia Fix Nitrogen. Applied and Environmental Microbiology. 71, 1:460 466

van de Broek A. \& J. Vanderleyden. 1995. Review: genetics of the Azospirillum-plant root association. Crit. Rev. Plant Sci. 14:445-466

Welller D.M. \& L.S. Thomashow. 1994. Current challenges in introducing beneficial microorganisms into the rhizosphere. Molecular ecology of rhizosphere microorganisms biotechnology and the release of GMOs. O'Gara F, Dowling DN, Boesten B, Winheim VCH (eds), Germany, 1-18

Zahir A.Z., M. Arshad \& W.T. Frankenberg. 2004. Plant Growth Promoting Rhizobacteria: Applications and Perspectives in Agricultura. Advances in Agronomy. 81:97-108 